\title{
INJECTIVE MODULES AND LOCALIZATION IN NONCOMMUTATIVE NOETHERIAN RINGS
}

\author{
BY
}

\section{ARUN VINAYAK JATEGAONKAR}

ABSTRACT. Let 8 be a semiprime ideal in a right Noetherian ring $R$ and $C(B)=\{c \in R \mid[c+8]$ regular in $R / Q\}$. We investigate the following two conditions:

(A) $C(B)$ is a right Ore set in $R$.

(B) $\mathcal{C}(\bar{B})$ is a right Ore set in $R$ and the right ideals of $R_{6}$, the classical right quotient ring of $R$ w.r.t. $C(Q)$, are closed in the $J\left(R_{6}\right)$-adic topology.

The main results show that conditions (A) and (B) can be characterized in terms of the injective hull of the right $R$-module $R / Z$. The $J$-adic completion of a semilocal right Noetherian ring is also considered.

Introduction. The well-known work of Matlis [12] makes a striking use of the localization and the completion to describe the injective modules over commutative Noetherian rings. It led us to examine the interaction between the injective modules and the localization in the context of noncommutative Noetherian rings.

Let $\&$ be a semiprime ideal in a right Noetherian ring $R$. Goldie's work in [2] shows that, in any attempt at defining the localization of $R$ at 8 , the set $\mathcal{C}(\bar{B})=$ $\{c \in R \mid[c+B]$ regular in $R / Q\}$ deserves special attention. In this connection, of particular interest is the condition (A): $\mathcal{C}(B)$ is a right Ore set in $R$. For, if (A) is satisfied then the usual procedure of clearing off the zero-divisors works and the classical right quotient ring $R_{8}$ of $R$ w.r.t. $\mathcal{C}(\bar{D})$ can be constructed.

Some preliminary results on the classical right quotient rings occupy $\S 1$. The main result of $\$ 2$ (Theorem 2.1) shows that if $(A)$ is satisfied then $R_{8}$ is a very tame ring indeed. It is semilocal; its Jacobson radical is the right ideal generated by the image of 8 ; further, $E_{R}(R / 8) \simeq E_{R_{8}}\left(R_{8} / J\left(R_{8}\right)\right)$. It is rather surprising that these results were known only under some severe conditions on $R$.

It is then natural to seek some useful characterizations of the condition (A). Lambek and Michler have shown (Theorem 5.6 of [10]) that (A) is satisfied if and

Received by the editors November 10, 1972.

AMS (MOS) subject classifications (1970). Primary 16A08, 16A46, 16A52; Secondary 16 A10.

Key words and phrases. Right Noetherian ring, localization at a semiprime ideal, classical localization, Ore set, AR-property, completion, injective modules. 
only if the generalized ring of right quotients of $R$ w.r.t. a certain torsion theory is well-behaved. (See Remark after Lemma 3.1.) Taking a completely different approach, we show in Theorem 3.2 that $(A)$ can be characterized in terms of certain conditions on $E_{R}(R / \not)$. The ramifications of this result will be discussed elsewhere; see [6].

In $\$ 4$, we consider the condition (B): $\mathcal{C}(\mathrm{B})$ is a right Ore set in $R$ and the right ideals of $R_{8}$ are closed in the $J\left(R_{8}\right)$-adic topology. The interest in (B) stems from the well-known Artin-Rees lemma. Assuming that $R$ is left as well as right Noetherian and $B$ is prime, Goldie [2] gave a criterion for (B) in terms of an ArtinRees type condition involving the symbolic powers of $B$. In Theorem 4.6 , we so recast Goldie's criterion that it applies to semiprime ideals in right Noetherian rings. However, the main results of $\$ 4$ (Theorems 4.5 and 4.7 ) are in a different direction. They show that condition(B) can also be characterized in terms of certain conditions on $E(R / \bar{B})$ and that, in the presence of $(B)$, the behavior of the $J\left(R_{6}\right)$-adic completion of $R_{8}$ is dictated by $E(R / 8)$. These results include some well-known results of Matlis [12].

The investigations of Goldie [2] and Lambek and Michler [10] are confined to prime ideals. It may be worthwhile to note that our emphasis on dealing with semiprime ideals is not solely motivated by a desire for generality. Given a prime ideal $p$ in a Noetherian ring $R$, our procedure of localization outlined in [6] requires us to examine $\mathcal{C}(\gamma(p))$ where $\gamma(p)$ is a certain semiprime ideal of $R$ constructed from $\nmid$. Even in orders over commutative Dedekind domains, one frequently encounters situations in which $\mathcal{C}(p)$ fails to be Ore from either side, $\gamma(p)$ fails to be a prime ideal but $\mathcal{C}(y(p))$ is Ore from both sides; see [6].

Some of the main results of this paper were announced in [6], [7].

1. Preliminaries. As usual, all rings, subrings and modules are assumed to be unitary.

In this section, we shall note some pertinent facts concerning left exact radicals and right Ore sets. Certain left exact radicals (equivalently, hereditary torsion theories or idempotent kernel functors) for $\bmod -R$ arising from the multiplicatively closed subsets of $R$ play an important role throughout the paper. However, only a superficial familiarity with left exact radicals is sufficient for our purpose and can be readily acquired from [4], [15].

Let $R$ be a ring and $\rho$ be a left exact radical for mod-R. The $\rho$-closure of a right ideal $K$ of $R$, denoted as $\rho$-cl $K$, is the unique right ideal of $R$ containing $K$ such that $(\rho-c l K) / K=\rho(R / K)$. Once we note that $\rho(M)$ is invariant under the action of End $M, M \in \bmod -R$, it becomes clear that the $\rho$-closure of a two-sided ideal of $R$ is a two-sided ideal of $R$. In particular, $\rho(R)=\rho-\mathrm{cl}(0)$ is a two-sided ideal of $R$. Let $\phi: R \rightarrow R / \rho(R)=\bar{R}$ be the canonical map. The restriction of $\rho$ 
to mod $-\bar{R}$ defines a left exact radical $\bar{\rho}$ for mod- $\bar{R}$. It is straightforward to check that, for every right ideal $K$ of $R, \rho-c l K=\phi^{-1}(\bar{\rho}-\mathrm{cl}(\phi(K)))$.

Let $\mathcal{D}$ be a multiplicatively closed subset of a ring $R$. For each $M \in \bmod -R$, let $\rho_{g}(M)$ be the unique largest submodule of $M$, each element of which is annihilated by some element of $\mathscr{D}$. Evidently, $\rho_{\mathscr{D}}$ is a left exact radical for mod-R. The left exact radical $\lambda_{\Phi}$ for $R$-mod is defined analogously. If $\lambda_{\Phi}-\mathrm{cl} t \subseteq \rho_{\Phi}-\mathrm{cl} t$ for all two-sided ideals $t$ of $R$ then $D$ will be called a right balanced subset of $R$.

A multiplicatively closed subset $D$ of $R$ is called a right Ore set if, for every $(d, r) \in \mathcal{D} \times R$, there exists $\left(d^{\prime}, r^{\prime}\right) \in \mathcal{D} \times R$ such that $d r^{\prime}=r d^{\prime}$. We allow zero-divisors to occur in the right Ore sets; even then, the useful right common multiple property holds, cf. p. 234 of [3]. Another useful result is the following folklore.

1.1. Proposition. The following conditions on a multiplicatively closed sub. set $\mathcal{D}$ of a ring $R$ are equivalent:

(1) $D$ is a right Ore set in $R$.

(2) For every $M \in \bmod R, \rho_{\mathscr{D}}(M)=\left\{\left.x \in M\right|_{R}(x) \cap \mathscr{D} \neq \varnothing\right\}$.

(3) For every $d \in \mathscr{D}, R / d R$ is $\rho_{\mathscr{D}}$-torsion.

(4) The image of $\mathfrak{D}$ in $R / P_{\mathscr{D}}(R)$ is a right Ore set.

Proof. Trivially, (2) $\Rightarrow(3) \Rightarrow(1) \Leftrightarrow(4)$. To see that $(1) \Rightarrow(2)$, use the right common multiple property.

A subset $X$ of a ring $R$ is a right regular subset if ${ }_{n}(x)=0$ for all $x \in R$. The left regular and regular subsets are analogously defined. Clearly, if $\mathscr{D}$ is a right Ore set in $R$ then its image in $R / \rho_{\Phi}(R)$ is a left regular right Ore set in $R / \rho_{\Phi}(R)$. This situation improves in the presence of a mild a.c.c.

1.2. Lemma. If a ring $R$ bas the a.c.c. on right annibilators then every left regular right Ore set in $R$ is regular.

Proof. See p. 229 of [3].

1.3. Proposition. Let $\mathfrak{D}$ be a right Ore set in a right Noetberian ring $R$. Then $\mathcal{D}$ is right balanced and its image $\bar{D}$ in $R / \rho_{\mathscr{D}}(R)$ is a regular right Ore set in $R / \rho_{D}(R)$.

Proof. That $\bar{D}$ is a regular right Ore set in $R / \rho_{g}(R)$ is immediate from 1.2. Let $\mathrm{t}$ be a two-sided ideal of $R$. The image $\mathscr{T}$ of $\mathscr{D}$ in $\widetilde{R}=R / t$ is a right Ore set; so, the image of $\tilde{D}$ in $\tilde{R} / \rho_{\mathcal{D}}(\tilde{R})$ is a regular set. Thus if $\tilde{x} \in \tilde{R}$ and $\tilde{d} \tilde{x}=0$ for some $\tilde{d} \in \widetilde{D}$ then $\tilde{x} \in \rho \tilde{\mathscr{D}}(\tilde{R})$. It follows that

$$
\lambda_{\mathscr{D}}(R / t)=\lambda_{\widetilde{D}}(\tilde{R}) \subseteq\left\{\tilde{x} \in \widetilde{R} \mid l_{\widetilde{R}}(\tilde{x}) \cap \widetilde{D} \neq \varnothing\right\} \subseteq \rho_{\widetilde{D}}(\tilde{R})=\rho_{\mathscr{D}}(R / t) .
$$


Consequently, $\lambda_{D^{-c l}} t \subseteq \rho_{D^{-c l}} t$.

If $\mathfrak{D}$ is a right Ore set in a right Noetherian ring $R$ then the classical right quotient ring of $R / \rho_{D}(R)$ w.r.t. the regular right Ore set $\mathbb{D}$ will be called the classical right quotient ring of $R$ w.r.t. $D$.

1.4. Proposition. Let $S$ be the classical right quotient ring of a right Noetberian ring $R$ w.r.t. a regular right Ore set $\mathcal{D}$. Then the following assertions bold:

(1) For every right ideal $F$ of $S$, the right ideal $R \cap F$ of $R$ is $P_{D}$-closed and $(R \cap F) S=F$.

(2) For every right ideal $K$ of $R, \rho_{g}-\mathrm{cl} K=R \cap K S$. The map $K \mapsto K S$ from the lattice of $\rho_{D}$-closed right ideals of $R$ to the lattice of right ideals of $S$ is an isomorphism whose inverse is defined by $F \mapsto R \cap F$. Consequently, $S$ is a right Noetberian ring.

(3) If $t$ is a two-sided ideal of $R$ then $S \mathrm{t} S=\mathrm{t} S$. A $\rho_{D}$-closed ideal $t$ of $R$ is a prime, resp. semiprime, if and only if the ideal t $S$ of $S$ is prime, resp. semiprime.

Proof. The first two assertions are well known; their routine proofs are omitted, cf. p. 234 of [3]. Since the third one is new, we provide some details.

Let $t$ be a two-sided ideal of $R$. Given $d \in \mathscr{D}$ and $t \in \mathrm{t}$, choose $d^{\prime} \in \mathcal{D}$ and $r^{\prime} \in R$ such that $d r^{\prime}=t d^{\prime} \in t$. As in the proof of 1.3, we have

$$
\rho_{\mathscr{D}}-\mathrm{cl} \mathrm{t} \supseteq\{x \in R \mid d x \in \mathrm{t} \text { for some } d \in \mathscr{D}\} \text {. }
$$

In particular, $r^{\prime} \in \rho_{D^{-}}$cl $t=R \cap t S$ which yields $d^{-1} t=r^{0} d^{0-1} \in(R \cap t S) S=$ $t S$. Hence $S t S=t S$.

Let $q$ be a prime ideal of $S$. If $t_{1}$ and $t_{2}$ are ideals of $R$ such that $R \cap q$ $\supseteq t_{1} t_{2}$ then $q=(R \cap q) S \supseteq t_{1} t_{2} S=\left(t_{1} S\right)\left(t_{2} S\right)$ since $t_{2} S=S t_{2} s$. It follows that $R \cap q$ is a prime ideal of $R$.

Let $p$ be a $\rho_{D}$-closed prime ideal of $R$. If $a_{1}, a_{2}$ are ideals of $S$ such that $a_{1} a_{2} \subseteq \not S$ then $\left(R \cap a_{1}\right)\left(R \cap a_{2}\right) \subseteq R \cap \not S=p$. It follows that $p S$ is a prime ideal of $S$.

The semiprime ideals are handled in a similar manner.

We finish this section with an observation on the right orders in semisimple rings which we could not locate in the literature.

1.5. Proposition. Let $R$ be a right order in a semisimple ring $Q$ and let $\rho$ be a left exact radical for mod- $R$ such that $\rho(R) \neq R$. Then $\rho(R) Q$ is a two-sided ideal of $Q, \rho(R)=R \cap \rho(R) Q$ and $R / \rho(R)$ is a right order in the semisimple ring $Q / \rho(R) Q$.

Proof. Let $d$ be a regular element of $R$ and $K=\{r \in R \mid d r \in \rho(R)\}$. Clearly, $K$ is a right ideal of $R$ and $d K \subseteq \rho(R) \subseteq K$; so $d K Q \subseteq \rho(R) Q \subseteq K Q$. Since $Q$ has 
the d.c.c. and $d$ is a unit in $Q$, we obtain $d K Q=\rho(R) Q=K Q$. Thus $d-1 \rho(R) Q=$ $\rho(R) Q$. Since $\rho(R)$ is a two-sided ideal of $R$, it follows that $\rho(R) Q$ is a two-sided ideal of $Q$.

Set $\mathrm{t}=R \cap \rho(R) Q$. Clearly, $\mathrm{t}$ is a two-sided ideal of $R$ and contains $\rho(R)$. Using the right common multiple property, we see that, for every $t \in t$, there exists a regular element $c$ of $R$ such that $t c \in \rho(R)$. Thus $\rho(R)$ is an essential right $R$-submodule of t. Choose a right ideal $L$ of $R$ such that $t \cap L=(0)$ and $t+L$ is essential in $R$. Then $\rho(R)+L$ is an essential right ideal of $R$; so, it contains a regular element of $R$, say $b$. Now, $t \simeq b \mathrm{t} \subseteq \mathrm{t} \cap(\rho(R)+L)=\rho(R)$ shows that $t$ is p-torsion. Hence $t=\rho(R)$. It is now straight forward to check that $R / \rho(R)$ is a right order in the semisimple ring $Q / \rho(R) Q$.

2. Classical localization. It is well known that if $R$ is a right order in a semisimple ring $Q$ then $R$ has only a finite number of minimal prime ideals, say $p_{1}, \cdots, p_{m}$; further, each $p_{i} Q$ is a maximal ideal of $Q, R \cap p_{i} Q=p_{i}$ and $(0)=$ $\bigcap_{i=1}^{m} p_{i}$. See [3].

Let 8 be a semiprime ideal in an arbitrary right Noetherian ring and $\pi: R \rightarrow$ $R / \&$ be the canonical map. Since $R / \&$ is a right order in a semisimple ring, as noted above, it has only a finite number of minimal prime ideals, say $\bar{p}_{1}, \cdots, \bar{p}_{m}$. The prime ideals $\pi^{-1}\left(\bar{p}_{1}\right), \cdots, \pi^{-1}\left(\bar{p}_{m}\right)$ are called the prime ideals associated with \&. Following Goldie [2], we define the symbolic powers of 8 as the members of the sequence $\left\{H_{n}(\mathrm{z}): n \in \mathrm{Z}^{+}\right\}$where $H_{1}(\mathrm{z})=8$ and

$$
H_{n+1}(z)=\lambda_{\xi(a)}-\mathrm{cl}\left(\rho_{\mathrm{C}(\mathrm{s})}-\mathrm{cl}\left(\mathrm{z} H_{n}(\mathrm{~B})\right)\right)
$$

for all $n \in \mathrm{Z}^{+}$. It is clear that $\mathcal{B}^{n} \subseteq H_{n}(\mathcal{B}) \subseteq 8$ and that each $H_{n}(z)$ is a two-sided ideal of $R$.

As usual, $J(R)$ denotes the Jacobson radical of a ring $R$. Recall that $R$ is semilocal (resp. quasi-local) if $R / J(R)$ is a semisimple (resp. simple Artinian) ring. The injective hull of a right $R$-module $M$ is denoted by $E_{R}(M)$.

We now describe the classical right quotient ring of $R$ w.r.t. $\mathcal{C}(z)$.

2.1. Theorem. Let $\&$ be a semiprime ideal of a right Noetberian ring $R$ such

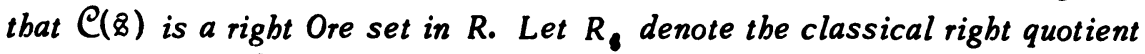
ring of $R$ w.r.t. $\mathcal{C}(8)$. Set $\bar{R}=R / \rho_{\mathrm{C}(s)}(R)$ and use bars to denote images in $\bar{R}$. Then the following assertions bold: $\mathbf{Z}^{+}$.

(1) $R_{b}$ is a semilocal right Noetherian ring with $J^{n}\left(R_{b}\right)=8^{n} R_{b}$ for all $n \epsilon$

If $p_{1}, \cdots, p_{m}$ are the prime ideals of $R$ associated with $\&$ then $\bar{p}_{1} R_{6}, \cdots$, $\bar{p}_{m} R_{8}$ are precisely the distinct maximal ideals of $R_{6}$. Consequently, $R_{6}$ is a quais-local ring if and only if $B$ is a prime ideal of $R$.

(2) For each $\left.n \in \mathrm{Z}^{+}, H_{n}(8)=\rho_{\mathrm{C}(6)}\right)^{-\mathrm{cl} 8^{n}}$ and $R / H_{n}(8)$ is a right order in the Artinian ring $R_{6} / J^{n}\left(R_{6}\right)$. 
(3) If $R$ is a semiprime ring then $\bar{R}$ and $R_{8}$ are semiprime rings.

(4) The injective bull of $R / Q$ in $\bmod -R$ is $R$-isomorphic with the injective bull of $R_{8} / J\left(R_{8}\right)$ in $\bmod \cdot R_{6}$.

Proof. Since every element of $\mathcal{C}(8)$ is a non-zero-divisor on $R / 8$ and every element of $\rho_{\mathcal{C}(8)}(R)$ is annihilated by some element of $\mathcal{C}(z)$, we get $\rho_{\mathcal{C}(8)}(R) \subseteq$ $\operatorname{ker}(R \rightarrow R / \bar{Q})=\overline{8}$; so, $\bar{C}(\overline{8})=\mathcal{C}(\overline{8})$. By $1.3, \mathcal{C}(\overline{8})$ is a regular right Ore set in $\bar{R}$. Hence $R_{B}$ exists and is a right Noetherian ring by 1.4 .

(1) Let $p_{1}, \cdots, p_{m}$ be the prime ideals of $R$ associated with $\&$. Since $\rho_{\mathcal{C}(8)}(R) \subseteq 8$, the prime ideals of $\bar{R}$ associated with $\overline{8}$ are $\bar{p}_{1}, \cdots, \bar{p}_{m}$. Let $\psi$ : $\bar{R} \rightarrow \bar{R} / \overline{8}$ be the canonical map, $Q$ be the semisimple classical right quotient ring of $\psi(\bar{R})$ and $\mathscr{D}$ be the right Ore set of the regular elements of $\bar{R}$. As noted before, $\psi\left(\bar{p}_{i}\right)=\psi(\bar{R}) \cap \psi\left(\bar{p}_{i}\right) Q$. We can express $\psi\left(\bar{p}_{i}\right) Q$ as a finite intersection of certain maximal right ideals of $Q$, say $\tilde{N}_{i 1}, \cdots, \widetilde{N}_{i k_{i}}$. Set $N_{i j}=\psi(\bar{R}) \cap \widetilde{N}_{i j}$ and $M_{i j}$ $=\psi^{-1}\left(N_{i j}\right)$. The lattice isomorphism indicated in 1.4 shows that each $N_{i j}$ is maximal among the $\rho_{\mathscr{D}}$-closed right ideals of $\psi(\bar{R})$. Consequently, each $M_{i j}$ is maximal among the $\rho_{C(\theta)}$-closed right ideals of $\bar{R}$. Using the lattice isomorphism of 1.4 again, it follows that each $M_{i j} R_{8}$ is a maximal right ideal of $R_{8}$. The right common multiple property of $\mathcal{C}(\bar{B})$ can be used now to see that

$$
J\left(R_{b}\right) \subseteq \bigcap_{i=1}^{m} \bigcap_{j=1}^{k_{i}}\left(M_{i j} R_{i}\right)=\bigcap_{i=1}^{m} \bar{F}_{i} R_{\mathrm{b}}=\overline{\mathrm{z}} R_{\mathrm{b}} .
$$

Let $F$ be an arbitrary maximal right ideal of $R_{\underline{B}}$ and $K=\bar{R} \cap F$. Then $\frac{K}{R}$

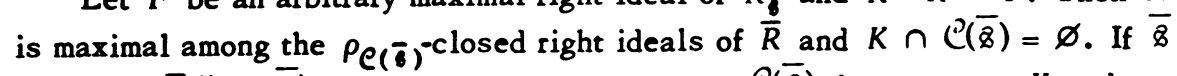
$\notin K$ then $\bar{R} /(K+\bar{z})$ is $\frac{\rho}{e(\bar{b})}$-torsion; so, $x+s \in \mathcal{C}(\bar{z})$ for some $x \in K$ and $s \in \bar{B}$. This yields $x \in K \cap \mathcal{C}(\bar{B})$, a contradiction! Thus $\bar{B} \subseteq K$; so, $\bar{B} R_{6} \subseteq K R_{6}=F$. It follows that

$$
J\left(R_{b}\right)=\bigcap_{i=1}^{m} \bigcap_{j=1}^{k_{i}}\left(M_{i j} R_{i}\right)=\bigcap_{i=1}^{m} \bar{p}_{i} R_{i}=\bar{B} R_{i}
$$

Since $J\left(R_{b}\right)$ has been expressed as a finite intersection of certain maximal right ideals of $R_{8}$, it follows that $R_{8}$ is semilocal. This, along with the fact that each $\bar{p}_{i} R_{b}$ is an ideal, shows that $\bar{F}_{1} R_{b}, \cdots, \bar{P}_{m} R_{b}$ are precisely the distinct maximal ideals of $R_{B}$. In particular, $R_{B}$ is quasi-local if and only if $\bar{Q}$ is prime. Since $R_{8} \overline{8} R_{b}=\overline{8} R_{b}=J\left(R_{6}\right)$, an induction gives $J^{n}\left(R_{8}\right)=\bar{b}^{n} R_{8}$ for all $n \in \mathrm{Z}^{+}$.

(2) Let us temporarily denote $\rho_{\mathcal{C}(6)}$ and $\rho_{C(\bar{b})}$ as $\rho$ and $\bar{\rho}$ respectively. The equality $J^{n}\left(R_{6}\right)=\overline{8}^{n} R_{6}$ along with 1.4 shows that $\bar{\rho}-\mathrm{cl} \bar{Q}^{n}=\bar{R} \cap J^{n}\left(R_{8}\right)$. This provides the following commutative diagram of ring homomorphisms 


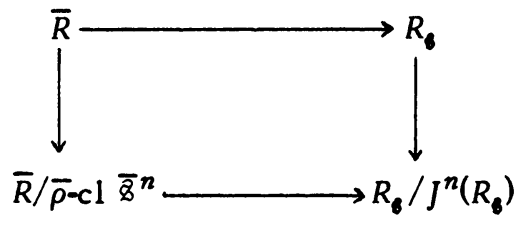

in which the vertical arrows are canonical surjections and the horizontal arrows are canonical injections. We shall identify $\bar{R} / \bar{\rho}-c 1 \bar{\delta}^{n}$ with the appropriate subring of $R_{8} / J^{n}\left(R_{b}\right)$. The ring $R_{b} / J^{n}\left(R_{8}\right)$ is semiprimary and right Noetherian, so right Artinian. Each element of it can be written as $\left[r+\bar{\rho}-c l \bar{B}^{n}\right]\left[c_{0}+\bar{\rho}-c l \bar{\delta}^{n}\right]^{-1}$ for some $c \in \mathcal{C}(\bar{Q})$ and $r \in \bar{R}$. It follows that $\bar{R} / \bar{\rho}-c l \bar{Q}^{n}$ is a right order in $R_{b} / J^{n}\left(R_{b}\right)$. It is easily seen that $\rho-\mathrm{cl} \bar{Q}^{n}$ is the full inverse image of $\bar{\rho}-\mathrm{cl} \overline{\mathrm{Q}}^{n}$ under $R \rightarrow \bar{R}$. Hence $R / \rho \propto \mathrm{cl} B^{n}$ is a right order in $R_{b} / J^{n}\left(R_{8}\right)$ for every $n \in \mathbf{Z}^{+}$.

We proceed to show that $\rho \circ \mathrm{cl} \bar{Z}^{n}=H_{n}(\not)$ for all $n \in \mathrm{Z}^{+}$. The equality is clear for $n=1$. Inductively, assume that it holds for $n$. We have $8^{n+1} \subseteq 8 H_{n}(8)$ which yields $\rho-c l \delta^{n+1} \subseteq \rho-c l\left(\delta H_{n}(\delta)\right) \subseteq H_{n+1}(8)$. On the other hand, for every $x \epsilon$ $8\left(\rho-c l \delta^{n}\right)$, there exists $c \in \mathcal{C}(8)$ such that $x c \in B^{n+1}$; by $1.1, x \in \rho-c 18^{n+1}$. In view of 1.3 , this yields $H_{n+1}(8) \subseteq \lambda_{\mathcal{C}(8)}-\mathrm{cl}\left(\rho-\mathrm{cl}\left(B^{n+1}\right)\right) \subseteq \rho-c l\left(\rho-c l\left(B^{n+1}\right)\right)=$ $\rho-\mathrm{cl} \mathrm{B}^{n+1}$. This completes the induction on $n$.

(3) Immediate after 1.5 .

(4) It is obvious that $R_{B}$ is the generalized ring of quotients of $R$ w.r.t. $\rho_{\mathrm{C}(8)}$ Let $\mathcal{F}: \bmod -R \rightarrow \bmod -R$ be the corresponding localization functor. Since $\mathcal{C}(\dot{B})$ is right Ore, $R_{8}$ is a flat left $R \cdot$ module and $\mathcal{F}=-\bigotimes_{R} R_{8}$. (See $\$ 13$ of [15].) Applying $\mathcal{F}$ to the exact sequence $0 \rightarrow \bar{B} \rightarrow \bar{R} \rightarrow \bar{R} / \bar{Q} \rightarrow 0$ of right $R$-modules, we see

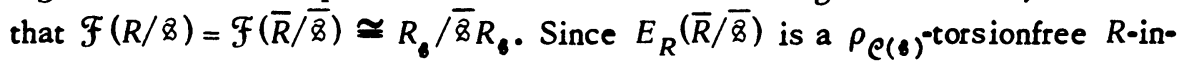
jective, it has a unique $R_{b}$-module structure extending the $R$-structure and as a $R_{8}$-module it is injective, cf. $\$ 8$ of [15]. From the construction of $\mathcal{F}$ as in [4], it is clear that $\bar{R} / \bar{B} \subseteq \mathcal{F}(\bar{R} / \bar{B}) \subseteq E_{R}(\bar{R} / \bar{Z})$. So $E_{R}(\bar{R} / \bar{B})$ is also the $R_{8}$-injective hull of $\mathcal{F}(\bar{R} / \bar{Q})$. The assertion follows.

Remarks. The salient part of (1), viz. $R_{8}$ is semilocal with $J\left(R_{8}\right)=\overline{\bar{z}} R_{8}$, is new even when 8 is prime. Lambek and Michler (Corollary 5.7 of [10]) obtain it only when $\delta$ is a maximal ideal in a right Noetherian ring satisfying a proper polynomial identity. It is inherent in Goldie's result [2] if $R$ is left as well as right Noetherian and 8 is a prime ideal satisfying condition (B). Part (2), although new, is inspired by [2]. We have not located any analogue of (3). The last part generalizes a result of Matlis [12] and is used in $\$ 4$ to generalize other results from [12].

3. The localizability criterion. A left exact radical $\rho$ for mod- $R$ is cogenerated by an injective right $R$-module $E$ if the following condition holds: A right $R$ module $M$ is $\rho$-torsionfree if and only if $M$ can be embedded in some direct product of copies of $E$. 
3.1. Lemma. If $B$ is a semiprime ideal in a right Noetherian ring $R$ then $\rho_{\mathrm{C}(\mathrm{b})}$ is cogenerated by the injective bull of the right $R \cdot$ module $R / 8$.

Proof. See [8].

It may be worthwhile to note that, using 2.1 and 3.1, Theorem 5.6 of [10] can be improved and made to hold for semiprime ideals.

We now prove the main result of this section.

3.2. Theorem. Let 8 be a semiprime ideal in a right Noetberina ring $R$. Then the following conditions are equivalent:

(1) $\mathcal{C}(8)$ is a right Ore set in $R$.

(2) If $N$ is a uniform right $R$-submodule of $R / 8$ and $M_{R}$ is a cyclic essential extension of $N$ such that $M / N$ is $\rho_{\mathrm{C}(6)}$-torsion then $M B=(0)$.

(3) Eacb element of $\mathcal{C}(8)$ acts as a non-zero-divisor on the injective bull of the right $R$-module $R / Z$.

Proof. (1) $\Rightarrow$ (3) is immediate from 2.1(4). The machinery used in its proof can be also employed to see that $(1) \Rightarrow(2)$. Thus, since $M / N$ is $\rho \mathrm{e}(6)^{\text {-torsion, }}$ we have $(M / N) \otimes_{R} R_{8}=(0)$; so, the canonical map $N \otimes_{R} R_{8} \rightarrow M \otimes_{R} R_{8}$ is an isomorphism. Since $N \otimes_{R} R_{6}$ embeds in $(R / 8) \otimes_{R} R_{8} \simeq R_{6} / \overline{8} R_{6}$, it follows that $\left(M \otimes_{R} R_{b}\right) \&=(0)$. But $M$ is a $\left.\rho_{C(6)}\right)^{\text {torsionfree module; so, it embeds }}$ in its module of quotients $M \otimes_{R} R_{B}$. Hence $M B=(0)$.

We proceed to show that $(2) \Rightarrow(1)$ and $(3) \Rightarrow(1)$. Assume for a moment that $R / d R$ is not $\rho_{\mathcal{C}(8)}$ torsion for some $d \in \mathcal{C}(\&)$. Using the a.c.c. in $R$, we can choose a proper right ideal $K$ of $R$ which is maximal among the proper $\rho e(6)^{-}$ closed right ideals containing $d R$. Set $M=R / K$. Using 3.1, we can obtain a uniform submodule $N$ of $M$ such that there exists a nonzero $R$-homomorphism $f: N \rightarrow R / 8$. If $\operatorname{ker} f \neq(0)$ then $M /$ ker $f$ contains a copy of the nonzero $\rho_{\mathrm{C}(6)}$ torsionfree module $f(N)$, contrary to our choice of $K$. A similar contradiction obtains unless $N$ is essential in $M$. After a change up to an isomorphism, we see that $M$ is a cyclic essential extension of the uniform submodule $N$ of $R / Z$ and $M / N$

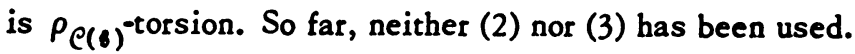

Now assume that condition (3) holds. Evidently, $d$ is a zero-divisor on $M$ and $M$ embeds in the injective hull of the right $R-$ module $R / 8$. Contradiction! In view of 1.1 , we get (3) $\Rightarrow(1)$.

Assume that condition (2) holds. Then $M B=(0)$ where $M$ is the module constructed above. Treated as a module over $R / 8, M$ is an essential extension of the nonsingular module $N$. So, $M$ is nonsingular over $R / 8$. But this is obviously false. Using 1.1 again, we see that $\mathcal{C}(\&)$ is right Ore.

Remark. Condition (2) allows us to decide whether $\mathcal{C}(8)$ is right Ore with- 
out forcing us to look very far in $E_{R}(R / 8)$. This is quite helpful in the applications. See [6].

Frequently, it so happens that we do have some right Ore set related with $\&$ which we would like to use to show that $\mathcal{C}(8)$ is right Ore. In such situations, the following result is of help. It also shows that the emphasis on $\mathcal{C}(q)$ in connection with the localization at 8 is not entirely misplaced. However, see [6].

3.3. Proposition. Let $\&$ be a semiprime ideal in a right Noetherian ring $R$. Then the following conditions are equivalent:

(1) $\mathcal{C}(8)$ is a right Ore set in $R$.

(2) There exists a right Ore set $\mathcal{D}$ in $R$ with $\rho_{\mathscr{D}}=\rho_{\mathcal{C}(8)}$.

(3) If $D$ is any multiplicatively closed subset of $R$ such that $\mathscr{D} \subseteq \mathcal{C}(\mathcal{B})$ and $P_{D}=\rho_{C(\theta)}$ then $D$ is right Ore in $R$.

Proof. Let $D$ be a right Ore set in $R$ with $\rho_{\mathscr{D}}=\rho_{\mathcal{C}(8)}$. If $r d \in \mathbb{8}$ with $r \in R$ and $d \in \mathscr{D}$ then $[r+8] d=[0+8]$; so, $[r+8] \in \rho_{\mathscr{D}}(R / \&)=(0)$. Thus, the image of $D$ in $R / \mathscr{B}$ is a left regular right Ore set in $R / \mathscr{Q}$. By 1.2 , we have $D \subseteq \mathcal{C}(\mathbb{Q})$.

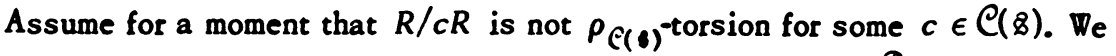
can choose a right ideal $K$ of $R$ maximally so that $c R \subseteq K$ and $\mathscr{D} \cap K=\varnothing$. Using 3.1, we obtain a right ideal $L \supsetneq K$ such that $L / K$ is isomorphic with a right $R$-submodule of $R / 8$. By our choice of $K, R / L$ has to be $\rho_{g}^{\text {-torsion; so }}$ $\mathscr{D} \cap L \neq \varnothing$. Choose $d_{1} \in \mathscr{D} \cap L, d_{2} \in \mathcal{D}$ and $r \in R$ such that $d_{1} r_{2}=c d_{2}$. Since $\mathscr{D} \subseteq \mathcal{C}(8),\left[r_{2}+8\right]$ is right regular in $R / \mathscr{Z}$. It follows that $r_{2} \in \mathcal{C}(\delta)$. Since elements of $\mathcal{C}(\&)$ are non-zero-divisors on $L / K$ and $[0+K]=\left[c d_{2}+K\right]=\left[d_{1}+K\right] r_{2}$, we a re forced to conclude that $d_{1} \in \mathcal{D} \cap K$. Contradiction! Using 1.1, we obtain $(2) \Rightarrow(1)$. The implications (3) $\Leftrightarrow(1) \Rightarrow$ (2) are trivial.

4. The right AR-property. In this section, we shall examine condition (B) stated in the introduction.

Recall that a two-sided ideal $t$ of a ring $R$ is said to have the right ARproperty if, for every right ideal $K$ of $R$, there exists $n \in \mathbf{Z}^{+}$such that $K \cap t^{n}$ $\subseteq K t$. The right AR-property is evidently a weakened form of the Artin-Rees property. Its connection with condition (B) is brought out by the following result which can be found in [13].

4.1. Theorem. If $R$ is a semilocal right Noetberian ring then the right ideals of $R$ are closed in the $J(R)$-adic topology if and only if $J(R)$ bas the right ARproperty.

Our immediate objective is to show that the equivalent properties stated in 4.1 can be characterized in terms of the socle series of $E(R / J(R))$. Recall that the socle series of a module $M$ is the ascending sequence $\left\{\operatorname{soc}_{n} M: n \geq 0\right.$ \} 
of submodules of $M$ defined inductively as follows:

$$
\operatorname{soc}_{0} M=(0) ; \quad \operatorname{soc}_{n+1} M=\pi_{n}^{-1}\left(\operatorname{soc}\left(M / \operatorname{soc}_{n} M\right)\right)
$$

where $\pi_{n}: M \rightarrow M /$ soc $_{n} M$ is the canonical map. As usual, if $X$ is a subset of a ring $R$ and $M$ is a right $R$-module then $\operatorname{ann}_{M} X$ denotes the set $\{m \in M \mid m x=0$ $\forall x \in X\}$. The routine proof of the following lemma is omitted.

4.2. Lemma. For every submodule $N$ of a right $R$-module $M$ and for every $n \geq 0$, we bave $\operatorname{soc}_{n} N=N \cap \operatorname{soc}_{n} M$; further, if $R$ is semilocal then $\operatorname{soc}_{n} M=$ $\operatorname{ann}_{M} J^{n}(R)$.

4.3. Proposition. The following conditions on an arbitrary ring $R$ are equivalent:

(1) If $M$ is a cyclic right $R \cdot$ module with essential socle then $M=\operatorname{soc}_{n} M$ for some $n \in \mathrm{Z}^{+}$.

(2) If $E$ is an injective right $R \cdot$ module with essential socle then $E=$ $\bigcup_{n \geq 0} \operatorname{soc}_{n} E$.

If $R$ is a semilocal ring then the above two conditions are equivalent to the following one:

(3) $J(R)$ bas the right AR-property.

Proof . (1) $\Rightarrow(2)$. Let $E$ be an injective right $R$-module with essential socle. If $M$ is any cyclic submodule of $E$ then soc $M=M \cap \operatorname{soc} E$ is essential in $M$; so, for some $n \in \mathbf{Z}^{+}$, we have $M=\operatorname{soc}_{n} M \subseteq \operatorname{soc}_{n} E$. Hence $E=\bigcup_{n \geq 0} \operatorname{soc}_{n} E$.

$(2) \Rightarrow(1)$. Let $M$ be a cyclic $R$-module with essential socle. Since $E_{R}(M)=$ $\bigcup_{n \geq 0} \operatorname{soc}_{n} E_{R}(M)$, it follows that for some $n \geq 0, M \subseteq M \cap \operatorname{soc}_{n} E_{R}(M)=\operatorname{soc}_{n} M$ by 4.2 .

We now assume that $R$ is a semilocal ring with Jacobson radical $J$.

$(1) \Rightarrow(3)$. Let $K$ be a right ideal of $R$ and $\psi: R \rightarrow E_{R}(K / K J)$ be an extension of the composite map $K \rightarrow K / K J \rightarrow E_{R}(K / K J)$. Evidently, $\psi(R)$ is a cyclic essential extension of its socle $K / K J$. By condition $(1), \psi(R)=\operatorname{soc}_{n} \psi(R)$ for some $n \in \mathbf{Z}^{+}$. Thus, by $4.2, \psi(R) J^{n}=(0)$ which yields $K \cap J^{n} \subseteq K \cap$ ker $\psi=K J$.

(3) $\Rightarrow(1)$. Let $M=R / L$ be a cyclic right $R$-module with essential socle $K / L, L \subseteq K \subseteq R$. We have a positive integer $n$ such that $K \cap J^{n} \subseteq K J$. Let $\pi$ : $R \rightarrow R / L$ be the canonical map. If $x$ is any element of $R$ such that $\pi(x) \in \pi(K)$ $\cap \pi\left(J^{n}\right)$ then $x=j+l_{1}=k+l_{2}$ for some $j \in J^{n}, k \in K$ and $l_{1}, l_{2} \in L$; clearly, $j \in K \cap J^{n} \subseteq K J \subseteq L$; so, $\pi(x)=0$. Thus, $\pi(K) \cap \pi\left(J^{n}\right)=(0)$. The assumed essentiality of $\pi(K)$ in $\pi(R)$ forces $0=\pi\left(J^{n}\right)=M J^{n}$. By 4.2, $M=\operatorname{soc}_{n} M$.

4.4. Corollary. The Jacobson radical of a semilocal right Noetherian ring $R$ bas the right AR-property if and only if the injective bull of the right R-module $R / J(R)$ is the union of its socle series. 
We are now in a position to prove our main result concerning condition (B).

4.5. Theorem. Let 8 be a semiprime ideal in a right Noetherian ring $R$. Then the following conditions are equivalent:

(1) $\mathcal{C}(8)$ is a right Ore set in $R$ and the Jacobson radical of $R_{6}$, the classical right quotient ring of $R$ w.r.t. $\mathcal{C}(8)$, has the right AR-property.

(2) If $M$ is a cyclic essential extension of a uniform right R-submodule $N$ of $R / B$, then $M Z^{n}=(0)$ for some $n \in Z^{+}$; further, if $M / N$ is $\rho_{e(0)}{ }^{-t o r s i o n}$ then $M B=$ (0).

(3) Let $E$ be the injective bull of the right $R \cdot \operatorname{module} R / 8$ and let $E_{n}=\operatorname{ann}_{E^{B^{n}}}$, $n \geq 0$. Then $E=\bigcup_{n \geq 0} E_{n}$ and each $E_{n+1} / E_{n}$ is nonsingular as a module over $R / 8$.

If these equivalent conditions are satisfied then $E$ can be canonically treat. ed as a right $R_{6}$-module and, as such, it is the injective bull of $R_{6} / J\left(R_{6}\right)$. Also, for each $n \geq 0$, the following equalities hold:

$$
\begin{aligned}
& \operatorname{soc}_{n} E_{R_{8}}=E_{n} ; \quad \operatorname{ann}_{E / E_{n}} \quad \&=E_{n+1} / E_{n} ; \\
& \operatorname{ann}_{R_{8}} E_{n}=J^{n}\left(R_{8}\right) ; \quad \operatorname{ann}_{R} E_{n}=H_{n}(8) .
\end{aligned}
$$

Proof. Assume that the condition (1) holds. We proceed to show that the condition (3) and the assertions stated after it hold.

Let $\phi: R \rightarrow \bar{R}=R / \rho_{\mathcal{C}(\theta)}(R)$ be the canonical map and $R$, be the classical right quotient ring of $R$ w.r.t. $\mathcal{C}(\bar{z})$. Set $\bar{z}=\phi(z)$ and $J=J\left(R_{8}\right)$. We shall repeatedly invoke Theorem 2.1. Thus, we already know that $E$ is the $R_{8}$-injective hull of $R_{8} / J$. Since $R_{b}$ is semilocal with $J^{n}=\overline{8}^{n} R_{6}$, it follows that $\operatorname{soc}_{n} E_{R_{b}}=\operatorname{ann}_{E} J^{n}=$ $\operatorname{ann}_{E^{2}} \overline{\bar{Q}}^{n}=E_{n}$ for all $n$. Now $E=\bigcup_{n \geq 0} E_{n}$ is immediate from 4.4. Since $R / \bar{Q}$ is a right order in $R_{6} / J$, the right $\left(R_{6} / J\right)$-module $E_{n+1} / E_{n}$ has to be nonsingular over $R / \bar{Q}$. Further, $E_{n+1} / E_{n}=\operatorname{soc}\left(E / E_{n}\right)_{R}=a n_{E} / E_{n} J=a n_{E} / E_{n}{ }^{Q}$. It remains to determine the annihilators of $E_{n}$. Using the fact that $E_{n}=$ ann $J^{n}$, it is easily seen that $E_{n}$ is the injective hull of $R_{b} / J$ in $\bmod -\left(R_{b} / J^{n}\right)$. Since $R_{b} / J^{n}$ is a right Artinian ring, its right socle is essẹntial in it. Consequently, for some $n \in Z^{+}$, the module $E_{n}^{(m)}$ contains a copy of the one-dimensional free right module over $R_{8} / J^{n}$. Hence ann $R_{R} E_{n}=J^{n}$ and $\operatorname{ann}_{R} E_{n}=\phi^{-1}\left(\operatorname{ann}_{\bar{R}} E_{n}\right)=\phi^{-1}\left(\bar{R} \cap \operatorname{ann}_{R}, E_{n}\right)$ $=\phi^{-1}\left(\bar{R} \cap J^{n}\right)=H_{n}(\overline{8})$.

We now show that $(2) \Rightarrow(1)$. It is clear from the latter half of condition (2) and Theorem 3.2 that $\mathcal{C}(\bar{B})$ is right Ore in $R$. Let $\bar{R}, \vec{z}, R_{8}$ and $J$ be as defined above. Let $M$ be a minimal right ideal of $R_{8} / J$. Since $R / B$ is a right order in $R_{d} / J$, it follows that $(\bar{R} / \bar{Q}) \cap M$ is a nonzero uniform right ideal of $\bar{R} / \bar{Q}$ with $E_{R_{B}}(M)$ as its $R$-injective hull. Thus, for any nonzero $x \in E_{R_{B}}(M)$, the cyclic $R$ submodule $x R$ is an essential extension of the uniform $R$-module $(\bar{R} / \bar{Q}) \cap M \cap x R$. 
So, for some $n \in \mathrm{Z}^{+}, 0=x \overline{8}^{n}=x \overline{8}^{n}=x J^{n}$. Now it is clear from 4.4 that $J$ has the right AR-property.

To complete the proof of the theorem, we show that $(3) \Rightarrow(2)$. Let $M$ be a cyclic essential extension of a uniform right $R$-submodule $N$ of $R / 8$. Since $M \subseteq$ $E=\bigcup_{n \geq 0} E_{n}$, it follows that $M 8^{n}=0$ for some $n \in \mathbf{Z}^{+}$. Now assume that $M / N$

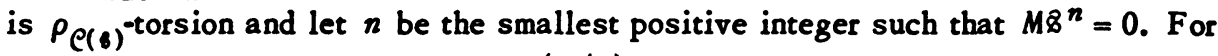
a moment, let $n>1$. Since $N \subseteq M \cap(R / 8) \subseteq M \cap E_{n-1}$, it follows that $\left(M+E_{n-1}\right) / E_{n-1} \cong M_{i}^{\prime}\left(M \cap E_{n-1}\right)$ is a nonzero $\rho_{\mathrm{C}(8)}$ torsion submodule of $E_{n} / E_{n-1}$ Contradiction!

The condition (3) and the assertions stated after it in Theorem 4.5 are related to Theorem 3.4 of [12].

We now use some of the ideas developed so far to recast Goldie's criterion for condition (B).

4.6. Theorem. A semiprime ideal $\&$ in a right Noetberian ring $R$ satisfies condition (B) if and only if, for each right ideal $K$ of $R$, there exists $n \in \mathrm{Z}^{+}$such that $R / H_{n}(8)$ is a right order in a right Artinian ring and $K \cap H_{n}(8) \subseteq \rho_{\mathrm{C}(8)}-\mathrm{cl}(K \overline{8})$.

Proof. Suppose that 8 satisfies condition (B). Let $\bar{R}, \bar{B}, R_{B}, J$ have their usual meaning and $\phi: R \rightarrow \bar{R}$ be the canonical map. As seen in $2.1, R / H_{n}(\mathscr{Z})$ is a right order in a right Artinian ring for all $n \in \mathrm{Z}^{+}$. Let $K$ be a right ideal of $R$. Set $\bar{K}=\phi(K)$. In view of 4.1 , we have $n \in \mathrm{Z}^{+}$such that $\left(\bar{K} R_{8}\right) \cap J^{n} \subseteq \bar{K} R_{8} J=$ $\bar{K} \bar{z} R_{8}$. Using 1.4 and 2.1 , we get

$$
\begin{aligned}
& K \cap H_{n}(8) \subseteq \rho_{\mathcal{C}(b)}-\mathrm{cl}(K) \cap \rho_{\mathcal{C}(8)^{-\mathrm{cl}}\left(8^{n}\right)} \\
& =\phi^{-1}\left(\bar{R} \cap \bar{K} R_{\mathrm{g}}\right) \cap \phi^{-1}\left(\bar{R} \cap J^{n}\right) \\
& \left.=\phi^{-1}\left(\bar{R} \cap \bar{K} R_{b} \cap J^{n}\right) \subseteq \phi^{-1}\left(\bar{R} \cap \bar{K} \bar{Q} R_{8}\right)=\rho_{\mathrm{C}(\mathrm{b})}\right)^{-\mathrm{cl}(K \bar{Q}) .}
\end{aligned}
$$

For the converse, we shall show that condition (2) of 4.5 holds. Let $M$ be a cyclic essential extension of a uniform $R$-submodule $N$ of $R / 8$. We may assume that $M=R / L$ and $N=K / L$ where $L \subseteq K$ are right ideals of $R$. Choose $n \in Z^{+}$ such that $R / H_{n}(8)$ is a right order in a right Artinian ring and $K \cap H_{n}(\&) \subseteq$

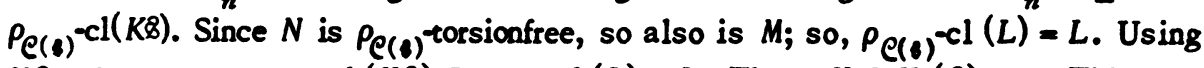
$N \delta=0$, we get $\rho_{\mathrm{C}(\delta)}-\mathrm{cl}(K \&) \subseteq \rho_{\mathrm{C}(8)}{ }^{-\mathrm{cl}}(L)=L$. Thus, $K \cap H_{n}(\&) \subseteq L$. This yields $H_{n}(8) \subseteq L$ since $K / L$ is essential in $R / L$. Since $\delta^{n} \subseteq H_{n}(8)$, it follows that $M B^{n}=(0)$.

Set $\tilde{R}=R / H_{n}(8)$ and $\tilde{B}=8 / H_{n}(\mathscr{B})$. Then $\tilde{B}$ is the prime radical of the right Noetherian ring $\tilde{R}$ and, by Small's theorem [14], $\mathcal{C}(\tilde{8})$ is a right Ore set in $\tilde{R}$. So,

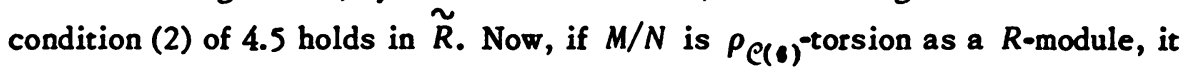


is $\rho_{\mathrm{C}(\tilde{B})}$-torsion as a $\tilde{R}$-module. Consequently, $M \tilde{Z}=(0)$ which yields $M \bar{Z}=(0)$. It follows that condition (B) is satisfied.

We note that if $B$ is a semiprime ideal in a left as well as right Noetherian ring $R$ then $R / H_{n}(8)$ is always an order in an Artinian ring; this can be readily seen from $\$ 4$ of [2]. Thus, in case 8 is prime and $R$ is left-right Noetherian, Theorem 4.6 reduces to Goldie's criterion, viz. Theorem 2.62 of [3].

Finally, we look at the J-adic completion of a semilocal right Noetherian ring, generalizing one more result of Matlis [12].

4. 7. Theorem. Let $R$ be a semilocal right Noetherian ring whose Jacobson radical $J$ bas the right AR-property. Let $E$ be the R-injective bull of the right $R$-module $R / J$ and $\hat{R}$ be the J-adic completion of $R$. Then $\hat{R}$ is canonically isomorphic with the biendomorphism ring of $E_{R}$. Further, $E$ can be canonically considered a right $\hat{R}$-module and, as such, it is $\Sigma$-injective if and only if $\hat{R}$ is right Noetherian.

Proof. Let $M$ be a right $R$-module such that $M=\bigcup_{n \geq 0} \operatorname{soc}_{n} M$. Let $x \in M$ and $\hat{r} \in \hat{R}$. By $4.2, x J^{k}=0$ for some $k \in \mathbf{Z}^{+}$. Choose a Cauchy sequence $\left\{r_{n}\right\}$ in $R$ which converges to $\hat{r}$. Then, for a sufficiently large $m, x r_{m}=x r_{n}$ for all $n \geq m$. Define $x \hat{r}=x r_{m}$. It is immediate that this makes $M$ a right $\hat{R}$-module. Further, if $\theta$ is a $R$-endomorphism of $M$ then $\theta(x \hat{r})=\theta(x) r_{n}$ for all $n \geq \mathrm{m}$ which yields $\theta(x \hat{r})=\theta(x) \hat{r}$. Thus $\theta$ is in fact a $\hat{R}$-endomorphism of $M$. If follows that biend $M_{R}$ = biend $M_{\hat{R}}$.

We now consider the $R$-module $E$. For $n \geq 0$, set $E_{n}=\operatorname{soc}_{n} E$ and $H_{n}=\{\theta \epsilon$ End $\left.E_{R} \mid \theta\left(E_{n}\right)=0\right\}$. Evidently, $E_{n}=\operatorname{ann}_{E} J^{n}$ and, due to 4.3, $E=\bigcup_{n \geq 0} E_{n}$. So, as shown above, $E$ has a canonical $\hat{R}$-module structure and biend $E_{R}=$ biend $E_{\hat{R}}$.

Let $n \geq 0$ and let $y \in E$ but $y \notin E_{n}$. By 4.2, we have $y R \cap E_{n+1} \neq y R \cap E_{n}$. Clearly, there is a nonzero $R$-homomorphism of $\left[\left(y R \cap E_{n+1}\right)+E_{n}\right] / E_{n}$ into $R / J$. This yields an element $b \in H_{n}$ such that $b(y) \neq 0$. So, $E_{n}=\operatorname{ann}_{E} H_{n}$ for all $n \geq 0$. It follows that $E_{n}$ is a biend $E_{R}$-submodule of $E$.

Now, let $b \in$ biend $E_{R}$. The restriction of $b$ to $E_{n}$ is a biendomorphism of $E_{n}$ as a right module over $R / J^{n}$. It is clear that $R / J^{n}$ is a right Artinian ring and $E_{n}$ is an injective cogenerator in $\bmod -\left(R / J^{n}\right)$. It follows that $\operatorname{ann}_{R} E_{n}=J^{n}$ and that the restriction of $b$ to $E_{n}$ is the right multiplication by some element $r_{n}$ of $R$; see [9]. The sequence $\left\{r_{n}\right\}$ is Cauchy in $R$. For, given $k \in \mathrm{Z}^{+}$, we have $E_{k}\left(r_{m}-r_{n}\right)=0$ for all $m, n \geq k$; this yields $r_{m}-r_{n} \in \operatorname{ann}_{R} E_{k}=J^{k}$. Let $\hat{r}$ be the limit of $r_{n}$. Then, for every $x \in E$, we have $x b=x \hat{r}$. So, the canonical map $\hat{R} \rightarrow$ biend $E_{R}$ is a surjection. Using Nakayama's lemma and the right AR-property of $J$, we have $\bigcap_{n \geq 0} J^{n}=(0)$; so, the natural map $R \rightarrow \hat{R}$ is an injection. Since $\operatorname{ann}_{R} E_{n}=J^{n}$ for $n \geq 0$, we see that $E_{R}$ is faithful; so $E_{\hat{R}}$ is faithful. Consequently, 
the canonical map $\hat{R} \rightarrow$ biend $E_{\hat{R}}=$ biend $E_{R}$ is an injection and so an isomorphism.

Assume that $\hat{R}$ is right Noetherian. By Theorem 2.7 of [13], the Jacobson radical $\hat{J}$ of $\hat{R}$ has the right AR-property. So, if $F$ denotes the $\hat{R}$-injective hull of $E_{R}$ then, by 4.3 , we have $F=\bigcup_{n \geq 0}$ ann $_{F} \hat{J}^{n}$. As a right $R$-module, $F$ splits into a direct sum of $E$ and a $R$-submodule $C$. Let $y \in C$. Then $y \hat{J}^{k}=0$ for some $k \in \mathbf{Z}^{+}$. Given $\hat{r} \in \hat{R}$, choose $r \in R$ such that $\hat{r}-r \in \hat{J}^{k}$. Then $y \hat{r}=y r \in C$. Thus, $C$ is in fact a $\hat{R}$-submodule of $F$. So, $C=(0)$; i.e., $E_{R}$ is an $\hat{R}$-injective and so a $\Sigma$-injective.

Conversely, suppose that $E_{R}$ is $\Sigma$-injective. Using 4.2 , it is easily seen that $\hat{R}$ satisfies condition (2) of 4.3. So, $\hat{J}$ has the right AR-property. By Theorem 2.7 of [13], $\hat{R}$ is a right Noetherian ring. Another proof of this part can be obtained from 9.B of [1].

The first part of the proof can be easily modified to show that, even when $J$ fails to have the right AR-property, $\hat{R}$ is canonically isomorphic with the biendomorphism ring of the right $R$-module $\bigcup_{n \geq 0} \operatorname{soc}_{n} E$.

We mention two open questions which are closely related to the matters considered here.

(1) Let $R$ be a semilocal Noetherian ring. Does $J(R)$ have the right AR-property? If $R$ is one-sided Noetherian, the example in [s] provides a negative answer since the right AR-property of $J(R)$ implies that $\bigcap_{n \geq 0} J^{n}(R)=(0)$. However, in the two-sided Noetherian case, the answer is not known even when $J(R)$ is assumed to have the left AR-property.

(2) Let $R$ be a semilocal (right) Noetherian ring whose Jacobson radical $J$ has the right AR-property. Is the J-adic completion of $R$ right Noetherian?

Acknowledgement. After the work on this paper was completed and the main results were privately circulated and announced [6], [7], the author received a preprint of [11] in which a part of Theorem 4.7 is proved by other methods. It also contains some new characterizations of condition (B).

\section{BIBLIOGRAPHY}

1. C. Faith, Modules finite over endomorphism rings, Lectures on Rings and Modules, Lecture Notes in Math., vol. 246, Springer-Verlag, Berlin and New York, 1972.

2. A. W. Goldie, Localization in non-commutative Noetherian rings, J. Algebra 5 (1967), 89-105. MR 34 \#7562.

3. - The structure of Noetherian rings, Lectures on Rings and Modules, Lecture Notes in Math., vol. 246, Springer-Verlag, Berlin and New York, 1972.

4. O. Goldman, Rings and modules of quotients, J. Algebra 13 (1969), 10-47. MR 39 $\# 6914$.

5. A. V. Jategaonkar, A counterexample in ring theory and homological algebra, J. Algebra, 12 (1969), 418-440. MR 39 \#1485. 
6. A. V. Jategaonkar, Injective modules and classical localization in Noetherian rings, Bull. Amer. Math. Soc. 79 (1973), 152-157.

7. - The AR-property and localization in Noetherian rings, Notices Amer. Math. Soc. 19 (1972), A751. Abstract \#72T-A274.

8. - The torsion theory at a semi-prime ideal (to appear).

9. T. Kato, Rings of U-dimension z 1, Tôhoku Math. J. (2) 21 (1969), 321-327. MR 40 \#1423.

10. J. Lambek and G. O. Michler, The torsion theory at a prime ideal in a right Noetherian ring, J. Algebra 25 (1973), 364-389.

11. - Completions and classical localizations of right Noetherian rings, Pacific J. Math. (to a ppear).

12. E. Matlis, Injective modules over Noetherian rings, Pacific J. Math. 8 (1958), 511528. MR 20 \#5800.

13. J. C. McConnell, The Noetherian property in complete rings and modules, J. Algebra 12 (1969), 143-153. MR 39 \#1487.

14. L. W. Small, Orders in Artinian rings, J. Algebra 4 (1966), 13-41. MR 34 \#199.

15. B. Stenström, Rings and modules of quotients, Lecture Notes in Math., vol. 237, Springer-Verlag, Berlin and New York, 1971.

DEPARTMENT OF MATHEMATICS, CORNELL UNIVERSITY, ITHACA, NEW YORK 14850 